\title{
The Growth Rate for the Number of Singular and Periodic Orbits for a Polygonal Billiard
}

\author{
A. Katok * \\ Department of Mathematics, California Institute of Technology, Pasadena, CA 91125, USA
}

\begin{abstract}
For any simply connected polygon in the plane, the number of billiard orbits which begin and end at a vertex grows subexponentially with respect to the length or to the number of reflections. This implies that the numbers of isolated periodic orbits and of families of parallel periodic orbits do grow subexponentially. The main technical device is a calculation showing that the topological entropy of the Poincare map for the billiard flow is equal to zero.
\end{abstract}

Let $\Delta \subset \mathbb{R}^{2}$ be a simply connected polygon. A broken (polygonal) line formed by the segments $\left[x_{0}, x_{1}\right],\left[x_{1}, x_{2}\right], \ldots,\left[x_{n-1}, x_{n}\right]$ will be called a generalized diagonal of $\Delta$ if it lies inside $\Delta$ except for the points $x_{0}, \ldots, x_{n}$, the points $x_{0}$ and $x_{n}$ are vertices of $\Delta$, the points $x_{1} \ldots x_{n-1}$ lie on the sides of $\Delta$, and for $i=1, \ldots, n-1$ the segments $\left[x_{i-1}, x_{i}\right]$ and $\left[x_{i}, x_{i+1}\right]$ form the same angle with the side of $\Delta$ passing through $x_{i}$ (cf. Fig. 1).

The total number of different generalized diagonals of $\Delta$ is always infinite. Let $D_{T}(\Delta)$ be the number of different generalized diagonals of $\Delta$ of length $\leqq T$.

The purpose of the first three sections of this note is to prove the following elementary geometric theorem:

Theorem. $\lim _{T \rightarrow \infty} \frac{\log \left(D_{T}(\Delta)\right)}{T}=0$.

Fig. 1

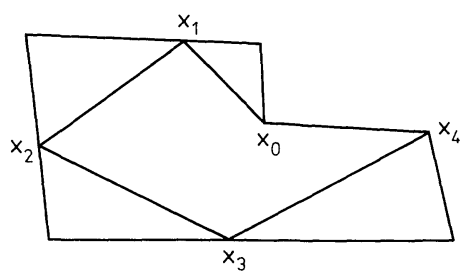

* Supported in part by NSF Grant \#DMS-8414400 
In Sect. 4 we derive some corollaries from this theorem and discuss generalizations and possible refinements of our results.

\section{Poincaré Map for the Billiard Flow}

Let $\left\{\varphi_{t}\right\}$ be the billiard flow defined on the set of all unit tangent vectors with the foot points in $\Delta$. Such a vector $u$ moves with unit speed along the straight line until it reaches the boundary of $\Delta$, then instantly changes direction according to the rule "the angle of incidence is equal to the angle of reflection," and continues along the new line. This motion is determined for all times unless the orbit hits a vertex of $\Delta$. Thus, generalized diagonals correspond to those orbits of the billiard flow in $\Delta$ which can be extended only for a finite time in both directions.

Let $\Gamma$ be the boundary of $\Delta$ and let $V$ be the set of all unit tangent vectors with foot points at $\Gamma$ directed inside $\Delta$. Let us denote by $\Phi$ the first-return map (Poincare map) induced by the billiard flow on $V$. This map and its iterates is defined, continuous and smooth everywhere except for the vectors whose billiard orbits hit vertices of $\Delta$.

Let us fix a vertex $P$ of $\Delta$ and an orientation on $\Gamma$. For $u \in V$ let $\theta$ be the angle between the positive direction on $\Gamma$ and the direction of $u$, and let $l$ be the length of the part of $\Gamma$, from $P$ to the foot point of $u$ in the positive direction. There is an ambiguity in defining the angle $\theta$ for the vector with the foot point in a vertex of $\Delta$, but this will not affect our considerations. We will use $\tau=\operatorname{cotan} \theta$ and $l$ as coordinates in $V$ so that $V$ is represented as a strip

$$
V=\{(\tau, l):-\infty<\tau<\infty, 0 \leqq l<L\},
$$

where $L$ is equal to the length of $\Gamma$.

Another convenient coordinate system is given by the coordinates $\theta$ and $s=l \sin \theta$. In $(\theta, s)$ coordinates we have

$$
V=\{(\theta, s): 0<\theta<\pi, 0 \leqq s<L \sin \theta\},
$$

so that $V$ has a natural compactification

$$
\bar{V}=\{(\theta, s): 0 \leqq \theta \leqq \pi, 0 \leqq s \leqq L \sin \theta\},
$$

and the map $\Phi$ preserves Lebesgue measure $d s d \theta=\sin \theta d l d \theta$. Parallel billiard orbits remain parallel after reflection about the same side of $\Delta$ and the distance between such orbits does not change after such a reflection. This means that near a point of continuity $\left(\theta_{0}, s_{0}\right)$ of $\Phi^{n}$, such that $\Phi^{n}\left(\theta_{0}, s_{0}\right)=\left(\theta_{1}, s_{1}\right)$, the map $\Phi^{n}$ has the following form:

$$
\Phi(\theta, s)=\left(\theta_{1}+\varepsilon\left(\theta-\theta_{0}\right), s_{1}+\varepsilon\left(s-s_{0}\right)+h(\theta)\right),
$$

where $\varepsilon= \pm 1$. The function $h$ is locally the same, but it changes during the passage through a discontinuity for $\Phi^{n}$. Thus, if $\Phi$ is continuous on a horizontal interval $\gamma=\left\{\theta=\theta_{0}, s_{1} \leqq s \leqq s_{2}\right\}$ the $s$-length of $\gamma, s(\gamma)=s_{2}-s_{1}$ is preserved by $\Phi$.

\section{Structure of Discontinuities of Poincaré Map}

The following construction is useful in many considerations concerning the billiard flows, including the proof of our theorem. 
Let us consider a billiard orbit starting from $\Gamma$. Instead of reflecting the orbit about a side of $\Delta$, we reflect the polygon about the same side and continue the orbit as a straight line. When this line meets a side of the reflected polygon we again reflect the polygon, etc. The sequence of reflections obtained that way depends on the orbit, but it is the same for all nearby orbits as long as they intersect the same sides of $\Gamma$. This process can be extended indefinitely along any orbit which does not hit a vertex of $\Gamma$. If it does, the straightened orbit will hit a vertex of one of the reflected polygons.

Let for $u \in V$ the associate line, $L(u)$, be the straight line parallel to $u$ and passing through the foot point of $u$. Let us call a set $B \subset V$ a bundle if the foot points of vectors from $B$ cover a segment on one of the sides of $\Delta$ and their associate lines are either all parallel or pass through the same point. The following lemma follows immediately from the definition.

Lemma 1. If the map $\Phi$ is continuous at all points of a bundle $B \subset V$, then $\Phi(B)$ is also a bundle.

A straightforward computation shows that in $(\tau, l)$ coordinates bundles are represented by line segments and vice versa. Thus, Lemma 1 immediately implies

Lemma 2. If the map $\Phi$ is continuous at every point of a segment $\gamma$ in $(\tau, l)$ coordinates, then $\Phi(\gamma)$ is also a segment.

Let us consider now the structure of discontinuities for the iterates of $\Phi$. As we mentioned above, the reflection process associated with a billiard orbit stops if the straightened orbit hits a vertex of the corresponding reflected polygon. Let us denote the point of $\mathbb{R}^{2}$ representing this vertex by $A$. Then if we move the foot point of the original vector along $\Gamma$, the billiard orbit whose associate line hits $A$ will move along a bundle in $V$. An endpoint of this bundle is the foot point of a vector $u$ whose associate line $L(u)$ directed toward $A$ meets another vertex in the process of reflection before $A$. We will call the segment representing this bundle and associated with a fixed sequence of reflections and a particular vertex $A$ of a reflected polygon, a branch of discontinuity and will denote it by $\beta_{A}$. There is a slight ambiguity in that notation because another admissible sequence of reflection may place the image of a vertex into the same point.

It is easy to see that the branch $\beta_{A}$ connects two other branches of discontinuity, in the case represented by Fig. $2, \beta_{B}$ and $\beta_{C}$. The vectors from $\beta_{A}$ represent singular points of a certain iterate $\Phi^{n}$ of $\Phi$. We will call the number $n$, the order of the branch $\beta_{A}$.

It is convenient to call the set of vectors with the foot point at a vertex of $\Delta a$ branch of discontinuity of order 0 . These points are also singular points for $\Phi$.

If the branch $\beta_{A}$ connects branches $\beta_{B}$ and $\beta_{C}$ as on Fig. 2, the order of $\beta_{A}$ is not less than the orders of $\beta_{B}$ and $\beta_{C}$. The pre-image $\Phi^{-1}\left(\beta_{A}\right)$ of any branch of discontinuity of order $n \geqq 1$ consists of several branches of discontinuity of order $n+1$. Replacing $\Phi$ by $\Phi^{-1}$ we define branches of discontinuity of negative order.

Lemma 3. Given $D>0$ and $\varepsilon>0$, there exists $N=N(D, \varepsilon)$ such that any branch of discontinuity of order $n,|n| \geqq N$ which passes through a point $\left(\tau_{0}, l_{0}\right)$, where $\left|\tau_{0}\right| \leqq D$ makes in coordinates $(\tau, l)$ an angle less than $\varepsilon$ with the horizontal direction. 
Fig. 2
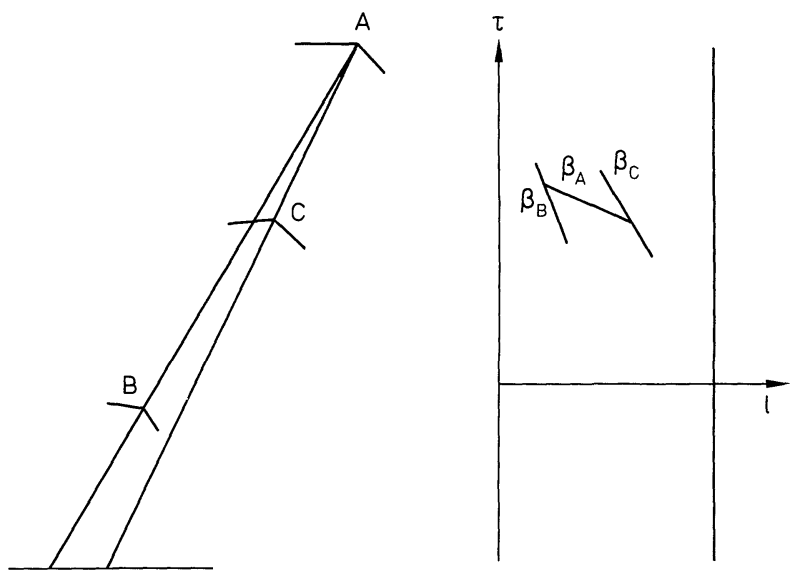

Proof. The coordinate representation of a branch $\beta_{A}$ passing through the point $u_{0}=\left(\tau_{0}, l_{0}\right)$ is

$$
\tau=\tau_{0}-\frac{l_{0}-l}{W}
$$

where $W=T \sin \theta_{0}, T$ is the distance from the foot point of $u_{0}$ to $A$. Since by the assumption the angle $\theta_{0}$ is bounded away from 0 and $\pi, W$ goes to infinity with $T$. But it is well known that $T>c_{1}|n|-c_{2}$, where $n$ is the order of the branch $\beta_{A}$ and $c_{1}, c_{2}$ are constants, because the number of successive reflections of a billiard orbit near a fixed vertex is bounded by a constant which depends only on the angle at that vertex. Thus, under the assumption of the lemma $W \rightarrow \infty$ as $|n| \rightarrow \infty$.

All branches of discontinuity of orders 0 and 1 divide the strip $V$ into a finite number of convex polygonal regions which we will call 1-cells. Convexity follows from the fact that the endpoints of every branch of order one are interior points of other branches of order 0 or 1 . The map $\Phi$ is defined and continuous inside each 1 cell so that by Lemma 2 the image of any segment inside an 1-cell is a segment. Actually, the map $\Phi$ inside each 1 -cell is a projective map in $(\tau, l)$ coordinates. Let us denote the partition of $V$ into 1-cells by $\zeta$. For a while we will speak about partitions of $V$ meaning partitions of the set of continuity for some iterate $\Phi^{n}$. In Sect. 3 the word "partition" will be given a slightly different meaning. Let

$$
\zeta_{n}=\zeta \vee \Phi^{-1} \zeta \vee \ldots \vee \Phi^{-n+1} \zeta \text {. }
$$

We will call elements of this partition $n$-cells. More generally, elements of the partition

$$
\Phi^{m-1} \zeta \vee \ldots \vee \Phi \zeta \vee \zeta \vee \Phi^{-1} \zeta \vee \ldots \vee \Phi^{-n+1} \zeta
$$

will be called $(m, n)$-cells.

Let $K$ be the total number of different branches of discontinuity of orders 0 and 1.

Lemma 4. Each $(m, n)$-cell $\sigma$ is a convex polygonal region with $\leqq K(m+n)$ sides. The maps $\Phi^{n}$ and $\Phi^{-m}$ restricted to $\sigma$ are continuous.

Proof. We will only give a proof for $n$-cells. The general case is completely similar. 
We proceed by induction in $n$. For $n=1$ the statement of the lemma follows from the definition of 1-cells. For any $n$-cell $\sigma$ we can find an $(n-1)$-cell $\sigma^{\prime}$ and an 1 cell $d$ such that

$$
\sigma=\sigma^{\prime} \cap \Phi^{-n+1} d .
$$

By the inductive assumption, $\sigma^{\prime}$ is a convex polygonal region with $\leqq K(n-1)$ sides. Since $\Phi^{n-1}$ is continuous on $\sigma^{\prime}$, it is a projective map so that $\Phi^{n-1} \sigma^{\prime}$ is also a convex polygonal region with the same number of sides. Since $d$ is also convex and has no more than $K$ sides, the intersection $\Phi^{n-1} \sigma^{\prime} \cap d$ is convex and has no more than $K n$ sides. Furthermore, $\Phi^{-n+1}$ is continuous on $\Phi^{n-1} \sigma^{\prime}$ so that $\sigma=\Phi^{-n+1}\left(\Phi^{n-1} \sigma^{\prime} \cap d\right)$ is also a convex polygonal region with $\leqq K n$ sides. The map $\Phi^{n}$ is continuous on $\sigma$ because $\Phi$ is continuous on $d$.

Remark. It is easy to see that the sides of any $n$-cell are branches of discontinuity of order at most $n$.

Lemma 5. Let $\sigma_{n}, n=1,2, \ldots$ be a sequence of embedded $n$-cells and let $\bar{\sigma}_{n}$ be the closure of $\sigma_{n}$ in $\bar{V}(c f .(1))$. Then the intersection $\gamma=\bigcap_{n=1}^{\infty} \bar{\sigma}_{n}$ is either a point or a
horizontal segment.

Proof. By Lemma 4, $\gamma$ is a closed convex set. Let us show that $\gamma$ cannot contain a non-horizontal interval. If $\gamma$ contains such an interval, then a parallel interval of the same length is contained inside an $n$-cell for $n=1,2,3, \ldots$. This will mean that the reflection process for all billiard orbits corresponding to a certain bundle of nonparallel vectors and of fixed size does not meet any obstacle, so that the sequence of reflections is the same for all those orbits. But this is impossible, because at every reflection about an image of a side of $\Delta$ an image of a vertex can be found at the end of the side, i.e. bounded distance away, whereas the cone generated by the orbits beginning from the initial bundle becomes arbitrary wide.

\section{Topological Entropy of Poincaré Map}

Let $C_{n}$ be the number of different $n$-cells. In this section we will prove the following proposition

Proposition. $\lim _{n \rightarrow \infty} \frac{\log C_{n}}{n}=0$.

Before proceeding to the proof of this proposition we will show how it implies the theorem about generalized diagonals. Any generalized diagonal containing $n-1$ reflections corresponds to the intersection of a branch of discontinuity of order 0 corresponding to the vertex $x_{0}$ and a branch of discontinuity of order $n$. Such an intersection is a vertex of an $n$-cell. By convexity (Lemma 4) each $n$-cell may contain not more than four vertices which belong to branches of discontinuity of order 0 . On the other hand, any generalized diagonal of length $T$ contains at most $K T$ reflections where $K$ is a constant. Thus

$$
D_{T}(\Delta) \leqq 4 C_{[K T]},
$$

so that the Proposition implies the Theorem. 
Proof of the Proposition. Let $N=\mathrm{Card} \zeta$ be the number of different 1-cells and let us denote those cells by $d_{1}, \ldots, d_{N}$. Let furthermore $V_{0} \subset V$ be the set of all points where all iterates $\Phi^{n}, n \in \mathbb{Z}$ are defined and continuous. Obviously, a point belongs to $V_{0}$ if and only if it lies in the interior of an $(n, n)$-cell for every positive integer $n$.

Let $\Omega_{N}=\{1, \ldots, N\}^{\mathbb{Z}}$ be the space of all doubly infinite sequences of symbols $1, \ldots, N$ with the product topology and let $S: \Omega_{N} \rightarrow \Omega_{N}$ be the left shift. The "coding" map $\psi: V_{0} \rightarrow \Omega_{N}$ is defined by

$$
\psi(x)=\left(\omega_{n}(x)\right)_{n \in \mathbb{Z}},
$$

where $\omega_{n}(x)$ is defined from $\Phi^{n} x \in d_{\omega_{n}(x)}$.

Since $\omega_{n-1}(\Phi x)=\omega_{n}(x)$, we have $\Phi \cdot \psi=\psi \cdot S$. Let $\Sigma \subset \Omega_{N}$ be the closure of the set $\psi\left(V_{0}\right)$ in the product topology. If $\omega=\left(\omega_{n}\right)_{n \in \mathbb{Z}} \in \Sigma$, then $\omega$ can be arbitrary well approximated by a point of the form $\psi(x), x \in V_{0}$ so that for every $n$,

$$
\bigcap_{k=-n}^{n} d_{\omega_{n}} \neq \phi .
$$

Let $\bar{d}_{k}$ be the closure of the 1 -cell $d_{k}$ in $\bar{V}$ [cf. (1)]. Thus, by (3) we have

$$
\sigma_{\omega} \stackrel{\text { def }}{=} \bigcap_{n=-\infty}^{\infty} \bar{d}_{\omega_{n}} \neq \phi .
$$

By Lemma 5 the set $\sigma_{\omega}$ is either a point or a horizontal segment.

Lemma 6. For every point $u \in \bar{V}$ there are at most countably many $\omega \in \Sigma$ such that $u \in \sigma_{\omega}$.

Proof. If $u \in V_{0}$ then $\omega$ is unique. If $u \in \bar{V} \backslash V_{0}$ then $u$ belongs to a branch of discontinuity. Let us first assume that $u$ is not one of the two infinity points corresponding to $\theta=0$ and $\theta=\pi$. Let us fix a positive integer $n$ and consider all branches of discontinuity of orders $k,|k| \leqq n$ passing through $u$. These branches divide a small neighborhood of $u$ into sectors belonging to different $(n, n)$-cells. Each cell corresponds to a word from $\{1, \ldots, N\}^{2 n-1}$ and if $u \in \sigma_{\omega}, \omega=\left(\omega_{n}\right)_{n \in \mathbb{Z}}$, the word $\left(\omega_{-n+1}, \ldots, \omega_{0}, \ldots, \omega_{n-1}\right)$ must coincide with one of the words represented by the cells. As $n$ increases, the new branches of discontinuity passing through $u$ become more and more horizontal (Lemma 3) so that for every $n$ the number of sectors not belonging to any fixed sector around the horizontal direction is uniformly bounded. This allows us to count all sectors appearing on all stages according to the angle with the horizontal direction.

If $u$ is an infinity point, let $\omega \in \Sigma$ and assume $u_{n} \in V_{0}$ and $u_{n} \rightarrow u$ in $\bar{V}$ and $\psi\left(u_{n}\right) \rightarrow \omega$. Then for any $m \in \mathbb{Z}, \psi\left(\Phi^{m} u_{n}\right) \rightarrow S^{m} \omega$. Assuming that $\omega$ is not a constant element, we have that a subsequence of $\Phi^{m} u_{n}$ converges to a point $u^{\prime} \in \bar{V}$ which is not an infinity point. For each $m$ there are only finitely many such $u^{\prime}$. We have $u^{\prime} \in \sigma_{S^{m_{\omega}}}$ so that for every given $u^{\prime}$ by the first case of the lemma, there are only countably many $\omega$ for which it is true. Thus for each $m, S^{m} \omega$ may only have countably many values and the lemma is proved.

Lemma 7. Every ergodic non-atomic shift-invariant measure $\mu$ on $\Sigma$ is supported by the set $\psi\left(V_{0}\right)$. 
Proof. Let $\Sigma_{0}=\left\{\omega \in \Sigma, \sigma_{\omega}\right.$ belongs to the interior of a 1-cell $\}$. Let us show that $\mu\left(\Sigma_{0}\right)=1$. If $\omega \in \Sigma \backslash \Sigma_{0}$, then $\sigma_{\omega}$, which is a point or a horizontal segment (Lemma 5), intersects a branch of discontinuity. If $\sigma_{\omega}$ is a segment, only its endpoints may belong to such a branch. If $\mu\left(\Sigma \backslash \Sigma_{0}\right)>0$ then by the ergodicity, $\mu$ almost every point $\omega$ visits the set $\Sigma \backslash \Sigma_{0}$ infinitely many times, i.e. there are infinitely many $n$ such that $S^{n} \omega \in \Sigma \backslash \Sigma_{0}$. If $\sigma_{\omega}$ is a point, that means that $\sigma_{\omega}$ belongs to more than one branch of discontinuity, but there are only countably many points in $\bar{V}$ with such a property. By Lemma 6, there are only countably many $\omega$ for which $\sigma_{\omega}$ may be one of those intersection points. Similarly, if $\sigma_{\omega}$ is a segment, one of its ends must belong to the same countable set and Lemma 6 applies again. Thus, the set of points visiting $\Sigma \backslash \Sigma_{0}$ infinitely many times (actually, more than once) is countable and since $\mu$ is a non-atomic measure, $\mu\left(\Sigma \backslash \Sigma_{0}\right)=0$.

Since $\psi\left(V_{0}\right)=\bigcap S^{n} \Sigma_{0}$ we have $\mu\left(\psi\left(V_{0}\right)\right)=1$.

Lemma 8. $\lim _{n \rightarrow \infty} \frac{\log C_{n}}{n}$ is equal to $h\left(\left.{ }^{S}\right|_{\Sigma}\right)$, the topological entropy of the shift $S$ restricted to the set $\Sigma$.

Let us construct a natural partition of $\Sigma$ corresponding to the partition $\zeta$ of $V_{0}$. Namely let

$$
c_{k}=\operatorname{clos} \psi\left(d_{k} \cap V_{0}\right), \quad k=1, \ldots, N .
$$

Sets $c_{k}$ are disjoint and form an open cover of $\Sigma$ which we will denote by $\xi$. The number of $n$-cells is equal to the number of elements in the cover

$$
\xi_{n}=\xi \vee S^{-1} \xi \vee \ldots \vee S^{-n+1} \xi \text {. }
$$

Since this cover is a topological generator for $S$, by definition

$$
h\left(\left.S^{S}\right|_{\Sigma}\right)=\lim _{n \rightarrow \infty} \frac{\log \operatorname{Card} \zeta_{n}}{n}=\lim _{n \rightarrow \infty} \frac{\log C_{n}}{n} .
$$

Lemma 9. For every Borel $S$-invariant ergodic measure $\mu$ supported by the set $\Sigma$ the entropy $h_{\mu}(S)$ is equal to zero.

Proof. Let us discuss the relationship between the partition $\zeta$ of $V_{0}$ and the partition $\xi$ of $\Sigma$. Since by Lemma $7 \mu\left(\psi\left(V_{0}\right)\right)=1$, from the measure-theoretic point of view $\xi$ can be viewed as a partition of the set $\psi\left(V_{0}\right)$. Let $\omega=\left(\omega_{n}\right)_{n \in \mathbb{Z}} \in \dot{\psi}\left(V_{0}\right)$ and let

$$
\sigma_{\omega}^{-}=\bigcap_{n=0}^{\infty} \Phi^{-n} d_{\omega_{n}}
$$

This set is an element of the partition $\zeta^{-}=\bigvee_{n=0}^{\infty} \Phi^{-n \zeta}$. Since the map is a one-to-one map between $V_{0}$ and its image, the set $\psi\left(\sigma_{\omega}^{-}\right)$coincides with the element of the partition $\xi^{-}=\bigvee_{n=0}^{\infty} S^{-n} \xi$ containing $\omega$.

To prove the lemma it is enough to show that up to a set of $\mu$-measure zero $S \xi^{-}=\xi^{-}$. For, since $\xi$ is by definition a generator for $S$, we have

$$
h_{\mu}(S)=h_{\mu}(S, \xi)=H\left(\left.{ }^{S \xi^{-}}\right|_{\xi^{-}}\right)=0 \quad[1] \text {. }
$$


By Lemma 5, the set $\sigma_{\omega}^{-}$is either a point or a horizontal interval. Since $\sigma_{S^{-1} \omega}^{-} \subset \Phi^{-1} \sigma_{\omega}^{-}$, then the set of $\omega$ for which $\sigma_{\omega}^{-}$is a point is $S$-invariant and by the ergodicity of $\mu$, this set either has full measure or has measure zero. In the former case $\zeta^{-}$is a partition into points so that $\xi^{-}$is also a partition into points, and since always $S \xi^{-} \geqq \xi^{-}$, we have $S \xi^{-}=\xi^{-}$. In the latter case let us consider the invariant transversal length $s\left(\sigma_{\omega}^{-}\right)$(cf. end of Sect. 1). Since $\Phi$ is continuous on $\sigma_{\omega}^{-}$, we have $s\left(\Phi \sigma_{\omega}^{-}\right)=s\left(\sigma_{\omega}^{-}\right)$. But since $S \xi^{-} \geqq \xi^{-}$, we have $\sigma_{S \omega}^{-} \supset \Phi \sigma_{\omega}^{-}$, so that $s\left(\sigma_{S \omega}^{-}\right) \geqq s\left(\sigma_{\omega}^{-}\right)$. Thus the function $s\left(\sigma_{\omega}^{-}\right)$does not decrease along the orbits of $S$. Since the measure $\mu$ is $S$ invariant this implies that $s\left(\sigma_{\omega}^{-}\right)$is $S$-invariant almost everywhere, and since $\mu$ is ergodic, $s\left(\sigma_{\omega}^{-}\right)=$const $\mu$-almost everywhere so that $\sigma_{S \omega}^{-}=\sigma_{\omega}^{-}$and $S \xi^{-}=\xi^{-}$.

Proof of the Proposition. By Lemma 8 and the variational principle for topological entropy (cf. e.g. [2, Sect. 18]) we have

$$
\lim _{n \rightarrow \infty} \frac{\log C_{n}}{n}=h\left(\left.S\right|_{\Sigma}\right)=\sup _{\substack{\text { supp } \mu \subset \Sigma \\ \mu \text {-ergodic }}} h_{\mu}(S) .
$$

By Lemma 9 the quantity at the right is equal to 0 . This finishes the proof.

\section{Concluding Remarks}

Our results allow us to estimate several other asymptotics related to a polygonal billiard. We will say that a certain quantity depending on a real parameter (as $D_{T}(\Delta)$ in the Theorem) or on a natural parameter (as $C_{n}$ is the Proposition) grows subexponentially if the assertion of the Theorem or the Proposition holds.

Corollary 1. The total number of branches of discontinuity of orders $k=0, \pm 1, \ldots, \pm n$ grows subexponentially as $n \rightarrow \infty$.

Proof. Follows immediately from Lemma 4 and the Proposition.

Any periodic orbit of the billiard flow in a polygon appears either as a member of a family of "parallel" orbits of the same length if the orbit contains an even number of links, or it is isolated and is surrounded by a family of parallel orbits of double length if the orbit has an odd number of links. The boundary of any family of parallel orbits consists of a number of generalized diagonals of the same or smaller length. Any generalized diagonal can appear as a part of the boundary for at most two different families of periodic orbits. Thus we associate with every isolated periodic orbit or a family of orbits of length $T$, a generalized diagonal of length $\leqq 2 T$, and no diagonal can appear in such a count more than four times. Thus, we have from the Theorem

Corollary 2. The total number of isolated periodic orbits and families of parallel periodic orbits of length $\leqq T$ grows subexponentially with $T$.

The subexponential asymptotics asserted by the Theorem, the Proposition, and the Corollaries can be generalized to the billiards in polyhedra of higher dimension. For an $m$-dimensional polyhedron $\Delta \subset \mathbb{R}^{m}$ we consider the number of continuous families of singular orbits starting on a given $k$-dimensional face, $k \leqq m-1$ ending on a given $l$-dimensional face $l \leqq m-2$ and containing an orbit of 
length $\leqq T$. All these numbers grow subexponentially. The generalized diagonals correspond to the case $k=l=0$. In the multi-dimensional case, periodic orbits in general do not appear in families. However, every periodic orbit corresponds to a sequence of reflections from the faces of the polyhedron which belongs to the compact part of the group generated by those reflections. Nearby orbits follow the same sequence of reflections and the boundary of the region is formed by several families of singular orbits of the above-mentioned type with $k+l=m-1$.

The scheme of the proof remains the same as for the two-dimensional case. It includes the construction of appropriate coordinate systems on the set of tangent vectors with the foot points on $\Gamma=\partial \Delta$ including Euclidean coordinates for the foot points on each face and projective coordinates for the tangent vector, the construction of branches of discontinuity and the piecewise-projective structure of the Poincare map, the coding and the description of elements of the partition $\zeta^{-}=\bigvee_{n=0}^{\infty} \Phi^{-n \zeta}$ as "horizontal" convex sets so that $\Phi$ acts isometrically on every such element in appropriate coordinates. We are planning to present the details for the multi-dimensional case in a separate paper.

The subexponential estimates for the asymptotics proved in this paper are weaker than the expected behavior of the quantities involved. It is easy to see that $D_{T}(\Delta)>$ const $\cdot T$. The quadratic asymptotic $D_{T}(\Delta) \sim c T^{2}$ which is obvious for the classical integrable billiards, i.e. for $\Delta$ being a rectangle, equilateral triangle or a right triangle with an angle $\frac{\pi}{6}$ or $\frac{\pi}{4}$, has been generalized by Gutkin [3] for a broader class of "almost integrable" billiards. For a rational polygon, i.e. a polygon all of whose angles are commensurable with $\pi$, a polynomial estimate for the growths of $D_{T}(\Delta)$ is known. Gutkin suggested the following elegant form of that estimate: $D_{T}(\Delta)<$ const $\cdot T^{g}$, where $g$ is the rank of the translation subgroup of the group generated by reflections about the sides of $\Delta$. He also pointed out that in the non-rational case this rank is infinite (personal communication). We conjecture that a polynomial estimate $D_{T}(\Delta)<c T^{k}$ holds for any polygon and similar estimates hold for all qualities described above for the polyhedral billiards.

For the polygons it is even possible that $k=2$ always, but we do not think that this is likely. The strongest evidence in favor of that conjecture is a recent result of Masur [7] who proved it for rational billiards. His result is based on Teichmüller theory considerations and on some previous results of Boshennitzan [4], who also showed that the quadratic estimate implies the main result of [5].

It is not known whether any polygonal billiard contains a periodic orbit. Recently Masur also using the methods of Teichmüller theory proved the existence of a periodic orbit for any rational polygon [6].

It is possible that for any rational polygon the total number $P_{T}(\Delta)$ of different parallel families of periodic orbits of length $\leqq T$ satisfies the inequality

$$
P_{T}(\Delta) \geqq \operatorname{const} D_{T}(\Delta) \text {. }
$$

On the other hand, we think that in the non-rational case typically

$$
\lim \sup \frac{\log P_{T}(\Delta)}{\log T}<\liminf \frac{\log D_{T}(\Delta)}{\log T} .
$$


Acknowledgements. I would like to thank Eugene Gutkin, Maciej Wojtkowski, and Boris Hasselblatt for useful discussions and for constructive criticism of the first draft of this paper.

\section{References}

1. Rochlin, V.A.: Lecture on the entropy theory of measure-preserving transformations. Russ. Math. Surv. 22, N5, 1-52 (1967)

2. Denker, M., Grillenberger, C., Sigmund, K.: Ergodic theory on compact spaces. Lecture Notes in Mathematics, Vol. 527. Berlin, Heidelberg, New York: Springer 1976

3. Gutkin, E.: Billiards on almost integrable polyhedral surfaces. Ergodic Theory Dyn. Syst. 4, 569-584 (1984)

4. Boshennitzan, M.: A condition for minimal interval exchange maps to be uniquely ergodic. Duke Math. J. 52, 723-752 (1985)

5. Kerckhoff, S., Masur, H., Smillie, J.: Ergodicity of billiard flows and quadratic differentials. Ann. Math. 124, 293-311 (1986)

6. Masur, H.: Rational billiards have periodic orbits, preprint

7. Masur, H.: The growth rate of trajectories of a quadratic differential. Ergodic Theory Dyn. Syst. (to appear)

Communicated by O. E. Lanford

Received December 26, 1984; in revised form February 27, 1987 